\title{
sequoia controls the type $1>0$ daughter proliferation switch in the developing Drosophila nervous system
}

\author{
Erika Gunnar, Caroline Bivik, Annika Starkenberg and Stefan Thor*
}

\begin{abstract}
Neural progenitors typically divide asymmetrically to renew themselves, while producing daughters with more limited potential. In the Drosophila embryonic ventral nerve cord, neuroblasts initially produce daughters that divide once to generate two neurons/glia (type I proliferation mode). Subsequently, many neuroblasts switch to generating daughters that differentiate directly (type 0 ). This programmed type $1>0$ switch is controlled by Notch signaling, triggered at a distinct point of lineage progression in each neuroblast. However, how Notch signaling onset is gated was unclear. We recently identified Sequoia (Seq), a C2H2 zinc-finger transcription factor with homology to Drosophila Tramtrack (Ttk) and the positive regulatory domain (PRDM) family, as important for lineage progression. Here, we find that seq mutants fail to execute the type $1>0$ daughter proliferation switch and also display increased neuroblast proliferation. Genetic interaction studies reveal that seq interacts with the Notch pathway, and seq furthermore affects expression of a Notch pathway reporter. These findings suggest that seq may act as a context-dependent regulator of Notch signaling, and underscore the growing connection between Seq, Ttk, the PRDM family and Notch signaling.
\end{abstract}

\section{KEY WORDS: Lineage tree, Cell cycle, Asymmetric division,} Combinatorial control, Notch

\section{INTRODUCTION}

During nervous system development, neural progenitor cells initially divide symmetrically to expand the progenitor pool. Subsequently, they switch to dividing asymmetrically to generate a lineage of neurons and glia (Gotz and Huttner, 2005; Sousa-Nunes and Somers, 2013). The generation of neurons and glia by each progenitor can occur by three basic modes: as directly differentiating neurons; via an intermediate daughter that divides once to generate two neurons/glia; or via a daughter that divides multiple times to generate several neurons/glia (Kriegstein et al., 2006; Fish et al., 2008; Franco and Muller, 2013). These three basic proliferation modes have been described in many animal species, and in Drosophila they are referred to as type 0, type I and type II, respectively (Bello et al., 2008; Boone and Doe, 2008; Bowman et al., 2008; Baumgardt et al., 2014; Bertet et al., 2014). The control of alternate daughter proliferation is of profound importance because it can act to expand the number of neurons/glia generated at a certain temporal stage in a developing lineage (Ulvklo et al.,

\footnotetext{
Department of Clinical and Experimental Medicine, Linkoping University, Linkoping SE-58185, Sweden.

*Author for correspondence (stefan.thor@liu.se)

iD S.T., 0000-0001-5095-541X
}

Received 18 May 2016; Accepted 22 August 2016
2012). It may furthermore act to control overall cell number in different regions of the developing nervous system, and might even act to modify its basic structure (Kriegstein et al., 2006; Fish et al., 2008; Nonaka-Kinoshita et al., 2013). However, in spite of being crucial for nervous system development, the genetic control of alternate daughter proliferation is not well understood.

In the developing Drosophila embryo, some 1200 neural progenitor cells [denoted neuroblasts (NBs)] generate the central nervous system (CNS) (Fig. 1A) (Bossing et al., 1996; Schmidt et al., 1997, 1999; Urbach et al., 2003, 2016; Wheeler et al., 2009; Birkholz et al., 2013). The CNS can be subdivided into the brain and the ventral nerve cord (VNC); here, we focus on the VNC. NBs undergo lineage progression by dividing asymmetrically to generate unique lineages of neurons/glia. Initially, NBs bud off daughter cells, denoted ganglion mother cells (GMCs), that divide once to generate two neurons/glia - the type I proliferation mode (Boone and Doe, 2008). Recently, studies have revealed that most, if not all, NBs subsequently switch from the type I mode into generating daughters that differentiate directly - the type 0 mode (Baumgardt et al., 2014). This type $\mathrm{I} \rightarrow 0$ (or I $>0$ ) switch is highly stereotyped and occurs at a distinct point in each lineage, but can vary considerably between lineages with respect to when it occurs during lineage progression (Fig. 1B). Previous studies of 21 cell cycle genes revealed that the precise regulation of four of these is instrumental for the type I $>0$ switch: Cyclin E (CycE), string (stg; Cdc25), E2f1

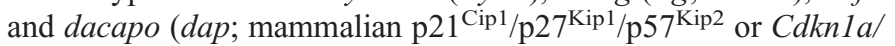
b/c) (Baumgardt et al., 2014; Bivik et al., 2015). In addition, several late-acting cues were identified that control the type $\mathrm{I}>0$ switch, including the late temporal genes castor (cas) and grainy head (grh), as well as the Hox gene Antennapedia (Antp). These regulators exert their roles by regulating the key cell cycle genes (Baumgardt et al., 2014). In addition, the canonical Notch pathway was also found to be important for the type $\mathrm{I}>0$ switch, via regulation of all four key cell cycle genes (Ulvklo et al., 2012; Bivik et al., 2015, 2016). Notch signaling is initially off in NBs - a prerequisite for establishment of NB cell identity in the neuroectoderm - but is gradually activated during lineage progression (Ulvklo et al., 2012; Bivik et al., 2016). The selective activation of Notch signaling in the latter stages of lineage progression is not, however, mediated by cas, grh or Antp (Ulvklo et al., 2012; Baumgardt et al., 2014; Bivik et al., 2016). Hence, it is unclear what controls the late temporal onset of Notch signaling in NBs to thereby ensure precise type $\mathrm{I}>0$ switches.

We recently conducted a screen for lineage development in the embryonic Drosophila CNS (Bivik et al., 2015). This screen focused on the thoracic NB5-6 lineage (NB5-6T) and was based on the expression of an FMRFa-eGFP transgene, reporting on the FMRFa neuropeptide-expressing neuron generated as the last cell in the NB5-6T lineage: a type 0 cell (Fig. 1B,C). This screen identified a number of regulatory genes acting to control NB5-6T lineage development, including the Notch pathway genes kuzbanian (kuz) 
A

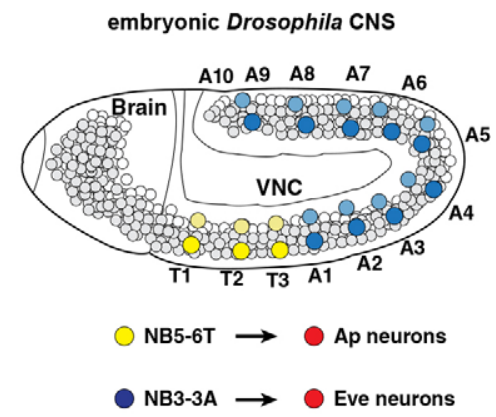

B
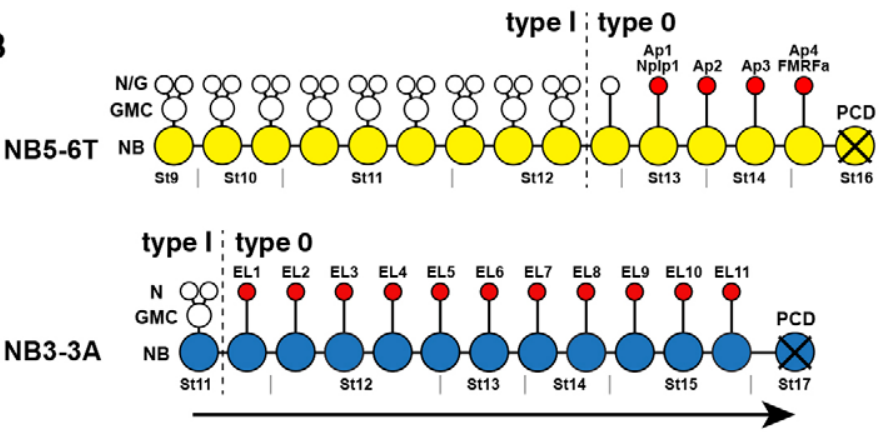


Fig. 1. sequoia mutants display extra cells in the NB5-6T and NB3-3A lineages. (A) During Drosophila embryogenesis, a single NB5-6T and NB3-3A forms in each thoracic and abdominal hemisegment of the ventral nerve cord (VNC), respectively. (B) NB5-6T starts dividing asymmetrically to renew itself and to bud off ganglion mother cells (GMCs) that divide once to create neurons (N) and/or glia (G) (Baumgardt et al., 2009), denoted the type I division mode (Boone and Doe, 2008). Subsequently, NB5-6T switches to the type 0 division mode: budding off daughter cells that differentiate directly into neurons (Baumgardt et al., 2009, 2014). The four last-born direct neurons, the Ap cells, can be identified by the expression of Eya, and the Ap1 and Ap4 neurons can further be uniquely identified by expression of the neuropeptides Nplp1 and FMRFa, respectively. At stage (St) 15, the NB5-6T exits the cell cycle, and at St16 it undergoes apoptosis (programmed cell death, PCD). NB3-3A displays a very short type I window and rapidly switches to a long type 0 window, generating directly differentiating neurons that are identifiable by Eve expression. NB3-3A exits the cell cycle at St16 and undergoes apoptosis at St17. (C,D) In a genetic screen scoring for FMRFa-EGFP expression, the mutant I(2R)O3FH was identified as lacking expression of FMRFa-EGFP, and was mapped to the sequoia (seq) gene (Bivik et al., 2015). (E) In control, the four Ap cells are identified by expression of Eya, and Ap1 by Nplp1 and Ap4 by FMRFa. (F,G) As anticipated from the loss of FMRFaEGFP expression in seq mutants, the expression of proFMRFa is also frequently lost. However, there are extra Ap cells generated, and often one extra Ap cell expressing Nplp1. $(H, I)$ When removing cell death $(H)$, even more cells are identified by Eya expression, showing that the effect of removing seq is partly masked by apoptosis of daughter cells. (J) Quantification of the number of cells expressing Eya, Nplp1 and FMRFa. (K,L) In seq mutants, there are fewer cells expressing Eve. (M) Removing cell death (ED225) in the seq mutant background results in additional cells. (N) Quantification of Eve cells. $(O, P)$ Expression of eg>GFP in control and seq mutant reveals additional cells in the lineage in seq mutants. (Q) Quantification of the number of cells expressing eg $>G F P$ in the NB3-3A lineage. ${ }^{*} P \leq 0.05,{ }^{* * *} P \leq 0.001$, one-way ANOVA with Bonferroni multiple comparison (J,N) or two-tailed Student's $t$-test (Q). $n \geq 16(\mathrm{~J}), n \geq 100(\mathrm{~N})$ or $n \geq 104(\mathrm{Q})$ lineages; error bars indicate s.d. AFT, air-filled trachea stage.

and neuralized (neur), as well as three of the four key cell cycle genes: dap, CycE and E2f1. Another interesting gene identified was the sequoia (seq) gene. seq was originally identified in a larval peripheral nervous system (PNS) screen (Gao et al., 1999; Brenman et al., 2001). The Seq protein contains two $\mathrm{C} 2 \mathrm{H} 2$ zinc fingers, with a high degree of primary sequence homology to the zinc-finger 
domain of the Drosophila Tramtrack (Ttk) protein, a member of the BTB-ZF family (Brenman et al., 2001). Both seq and $t$ tk have previously been linked to the Notch pathway (Guo et al., 1996; Brenman et al., 2001; Althauser et al., 2005; Jordan et al., 2006; Andrews et al., 2009). Comparing with mammalian proteins, Seq is most homologous to Prdm10, a member of the positive regulatory domain (PRDM) family, many of which are involved in embryonic development and Notch signaling (Hohenauer and Moore, 2012; Zannino and Sagerstrom, 2015). Because of the connections between seq, $t t k$, the PRDM family and Notch signaling, we pursued the role of seq with the aim of gaining further insight into the temporal activation of Notch signaling required for precise type $\mathrm{I}>0$ daughter switches.

We find that seq mutants display extensive aberrant proliferation that extends into late embryogenesis, a stage at which wild-type embryos do not display any dividing cells. Detailed single-lineage analysis, together with global VNC analysis, reveals that seq mutants frequently fail to undergo the type I $>0$ daughter switch. In addition, seq mutants show increased NB proliferation. Cell cycle protein expression analysis reveals that seq mutants show elevated expression of $\mathrm{CycE}$ and E2f1. Seq protein expression gradually declines in NBs, and seq mutants show elevated and premature Notch pathway activation. Genetic analysis further reveals strong genetic interaction between seq and the Notch pathway. These results suggest that seq plays an important temporal role in controlling Notch signaling in order to ensure precision in the type $\mathrm{I}>0$ daughter proliferation switch. These findings underscore the growing appreciation of the importance of the seq, ttk and PRDM regulators as modulators of the Notch pathway.

\section{RESULTS \\ seq mutants display increased cell numbers in NB5-6T and NB3-3A}

We recently identified a programmed daughter cell proliferation switch in many Drosophila embryonic VNC lineages (Baumgardt et al., 2014). We found that most, if not all, lineages start out by generating daughters that divide once to generate two neurons/glia the type I proliferation mode - but subsequently switch to generating daughters that differentiate directly into neurons - the type 0 proliferation mode. Two useful model lineages for studying the type I $>0$ switch are the NB5-6T and NB3-3A lineages (Fig. 1A). NB5-6T has a long type I window, generating nine dividing daughters, followed by a shorter type 0 window, generating five directly differentiating neurons (Baumgardt et al., 2014). The last four neurons born in this lineage are the Apterous neurons, expressing the LIM-homeodomain protein Apterous (Ap) and the transcriptional co-factor Eyes absent (Eya). The four Ap neurons can be further subdivided by the selective expression of the Nplp1 and FMRFa neuropeptides in the Ap1 and Ap4 cells, commencing at a late embryonic stage [air-filled trachea (AFT)] (Fig. 1B) (Baumgardt et al., 2007). In contrast to NB5-6T, NB3-3A displays a short type I window and immediately switches to a long type 0 window, generating a series of directly differentiating neurons that can be identified by expression of the transcription factor Evenskipped (Eve) (Fig. 1B) (Baumgardt et al., 2014). NB5-6T and NB3-3A exit the cell cycle at stage (St) 15 and St16, respectively, then die by programmed cell death (PCD) (Baumgardt et al., 2009, 2014; Karlsson et al., 2010).

The restricted expression of FMRFa to the Ap4 neuron formed at the end of the NB5-6T lineage previously allowed us to use an FMRFa-eGFP transgene as a potent marker for lineage progression in an EMS forward genetic screen. This screen resulted in the identification of several mutants that displayed proliferation defects in the NB5-6T lineage, including one allele for the seq gene: $\operatorname{seq}^{03 F H}$ (Fig. 1C,D) (Bivik et al., 2015). We analyzed the role of seq in NB5-6T at stage AFT using our seq ${ }^{03 F H}$ allele, the seq vr5-5 allele (a nonsense mutation) (Brenman et al., 2001) and a deletion for the region: $s^{D} q^{D f}=D f(2 R)$ Exel8057. seq ${ }^{03 F H}$ and $s e q^{v r 5-5}$ were of similar strength (Fig. 1J), and throughout subsequent studies we used the $s e q^{v r 5-5} / s^{D f}$ allelic combination.

We found that while seq mutants often displayed loss of FMRFa, we observed additional Ap neurons, identified by Eya staining, and frequently one additional Ap neuron expressing the Nplp1 neuropeptide (Fig. 1E-G,J). To address whether even more Ap neurons are generated but undergo PCD, we combined seq with a genomic deletion blocking PCD in the developing VNC $[D f(3 L)$ ED225]. Although ED225 does not generate extra Ap cells (Baumgardt et al., 2014), we found that seq, ED225 double mutants displayed additional Ap cells beyond those observed in $s e q$ mutants alone (Fig. 1H-J).

Next, we analyzed the generation of cells in the NB3-3A lineage. Using Eve as marker for neurons in this lineage, we observed a minor reduction of Eve-expressing cells in seq mutants (Fig. 1K-L,N). This was surprising given that we observed additional Eyaexpressing cells in NB5-6T in seq mutants. Similar to the analysis of NB5-6T, to address whether potential supernumerary cells in the NB3-3A lineage undergo PCD we analyzed Eve cell numbers in ED225 single and seq;ED225 double mutants. However, we only observed minor changes in Eve cell numbers in these genotypes (Fig. 1M,N). Hence, rather than being removed by PCD we argue that potential supernumerary cells in the NB3-3A lineage might not activate Eve, in particular if they result from aberrant division of type 0 daughters, which would likely be asymmetric in nature and might result in activation of Eve in only one of the aberrant daughter cells. To test this, we introduced the eg-Gal4/UAS-GFP marker - a transgenic reporter specifically expressed in NB3-3A - into seq mutants and quantified the total number of cells in the lineage. This now revealed supernumerary cells in NB3-3A in seq mutants, with an average increase of 3.5 cells/lineage (Fig. 1O-Q).

We conclude that seq mutants display supernumerary cells in both NB5-6T and NB3-3A. Generation of supernumerary cells is partly masked by PCD in NB5-6T and by failure to turn on the specific postmitotic marker Eve in NB3-3A daughter cells.

\section{seq mutants fail to execute the type $\mid>0$ programmed daughter proliferation switch in both NB5-6T and NB3-3A}

To identify the mechanism underlying the generation of supernumerary cells in the NB5-6T and NB3-3A lineages we analyzed cell divisions over time using antibodies that detect phosphorylated Ser10 on Histone H3 ( $\mathrm{pH} 3$ ), Prospero (Pros) and Deadpan (Dpn) to distinguish dividing NBs ( $\mathrm{pH} 3$ positive, Pros cortical, Dpn nuclear) from dividing daughters ( $\mathrm{pH} 3$ positive, Pros nuclear, Dpn negative) (Baumgardt et al., 2014).

Analyzing NB5-6T first, we observed a high percentage of dividing NBs and daughters at early stages in the control, followed by rapid decline down to zero divisions, first in daughters and then in NBs (Fig. 2A,C,D). This is in line with the programmed type I $>0$ switch in NB5-6T (Fig. 2I) (Ulvklo et al., 2012; Baumgardt et al., 2014). By contrast, in seq mutants we observed a tendency for elevated rates of NB divisions at St15-16, and significant elevation of daughter divisions at St14 (Fig. 2A-D). In addition, there was a striking and significant extension of daughter divisions into St15-17 - stages at which control embryos never display any daughter divisions (Fig. 2B-D). 



\section{Control}

seq $^{\text {vr5-5}} /$ seq $^{\text {Df }}$
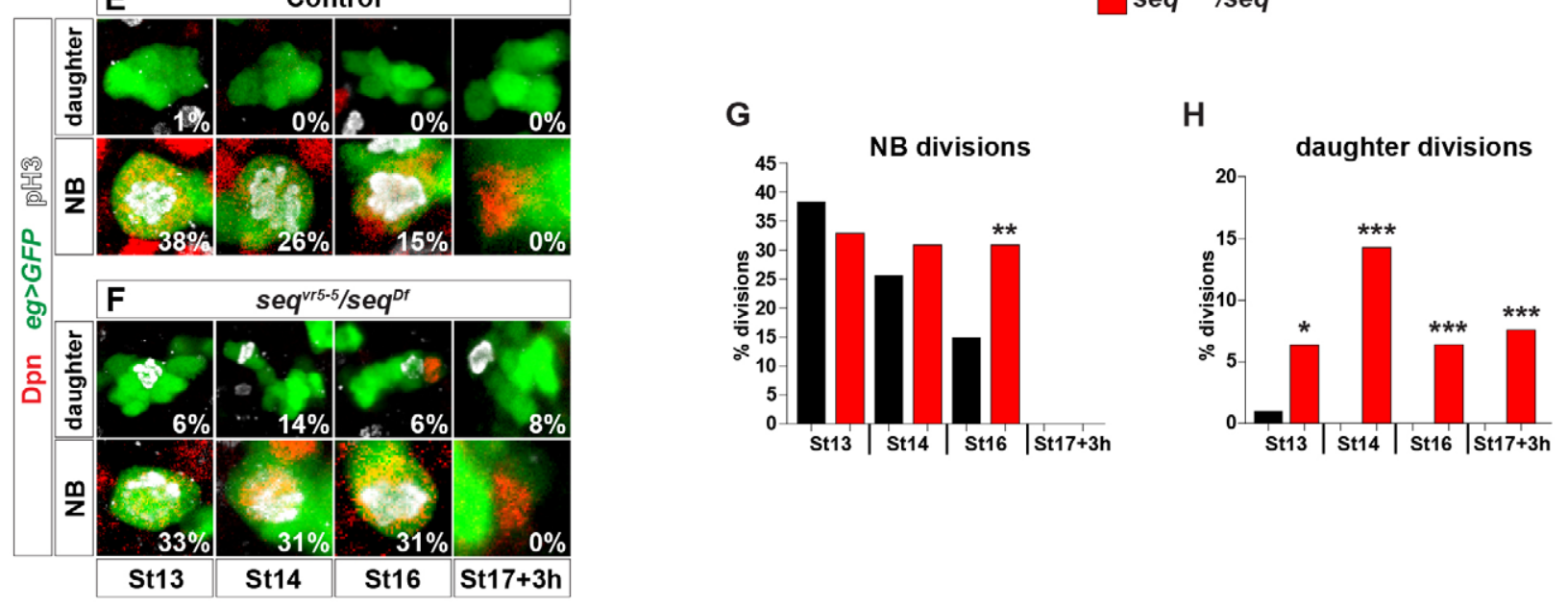

I
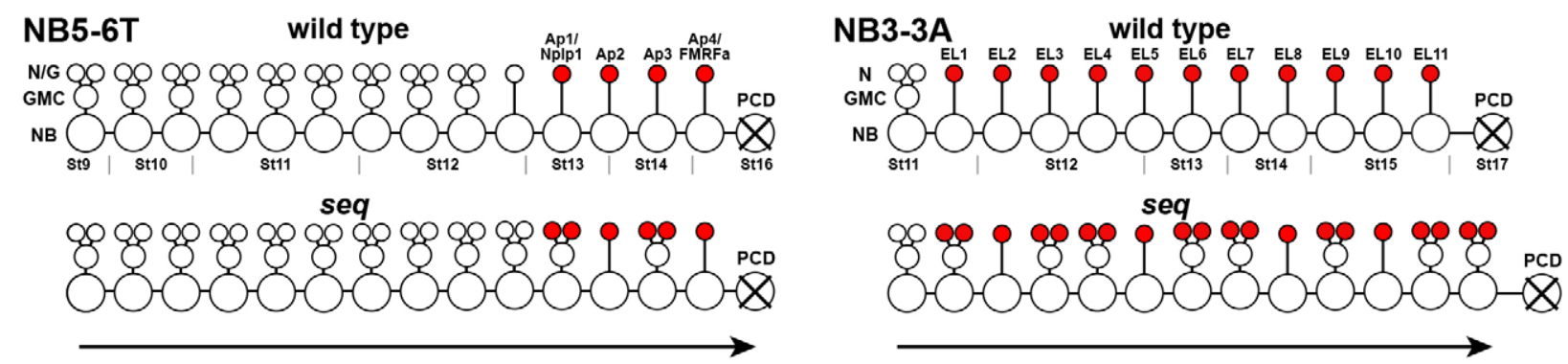

Fig. 2. seq mutants display overproliferation in the NB5-6T and NB3-3A lineages. (A,B) Divisions in the NB5-6T lineage. (A) In control, there is a decline in the number of divisions in the NB5-6T lineage over time. The NB divides until St16, while daughter divisions are only observed until St14. (B) In seq mutants, although the NB does not divide for longer, daughter divisions are extended in time and are also elevated at St14. (C,D) Quantification of NB and daughter divisions in the NB5-6T lineage. (E,F) Divisions in the NB3-3A lineage. (E) In control, daughter divisions rapidly decline and are not detected after St13, while NBs divide until St16. (F) In seq mutants, although the NB does not divide for an extended time, the number of divisions is elevated at St16. In addition, aberrant daughter divisions are observed at all stages analyzed. $(\mathrm{G}, \mathrm{H})$ Quantification of NB and daughter divisions in the NB3-3A lineage. ${ }^{*} P \leq 0.05,{ }^{* *} P \leq 0.01,{ }^{* * *} P \leq 0.001$, Chi-square test; $n \geq 60(\mathrm{C}, \mathrm{D})$ or $n \geq 35(\mathrm{G}, \mathrm{H})$ lineages. (I) Diagram illustrating the failure to execute the type $1>0$ switch in seq mutant NB5-6T and NB3-3A. By contrast, NB exit appears to occur at the correct stage.

Next, we analyzed NB3-3A. As previously described (Baumgardt et al., 2014), in control we observed a high percentage of NB divisions at St13-15, while daughters only displayed an occasional division at St13 and no subsequent divisions (Fig. 2E). These findings are in line with the early type I>0 switch in NB3-3A (Fig. 2I) (Baumgardt et al., 2014). In seq mutants, we observed significantly elevated NB divisions at St16 and elevated daughter divisions at St13, as well as aberrant daughter divisions extending from St14 into St17 (Fig. 2E-H).

From these results we conclude that the supernumerary cells observed in both the NB5-6T and NB3-3A lineages (Fig. 1) primarily result from a failure to execute the programmed type $\mathrm{I}>0$ daughter proliferation switch, although extended NB proliferation may also contribute to the increased cell number with regards to NB3-3A (Fig. 2I). 
seq mutants fail to execute the type I>0 programmed daughter proliferation switch globally in the VNC

The expression of seq RNA and Seq protein throughout the entire developing Drosophila VNC (Brenman et al., 2001) and the proliferation effects observed in two different NB lineages prompted us to address the role of seq globally in the VNC. To this end we again relied upon the combination of $\mathrm{pH} 3$, Pros and Dpn to help distinguish between dividing NBs and daughters in the VNC (Fig. 3A) (Baumgardt et al., 2014). In controls, there was a gradual decrease in both NB and daughter divisions, evident from St12 to St14, in both thorax (T2-T3) and abdomen (A1-A2) (Fig. 3C-F). In seq mutants, both NB and daughter divisions were significantly elevated at St13 and St14 in thorax and abdomen (Fig. 3B-F).

The elevated proliferation observed at St13-14 raised the possibility that proliferation might continue abnormally throughout the entirety of embryonic development. It is well established that all NBs in the developing VNC stop generating daughters during St12-17, after a stereotyped NB-specific schedule (Schmidt et al., 1997; Schmid et al., 1999; Rogulja-Ortmann et al., 2007; Karlsson et al., 2010). In line with this notion, control embryos at late embryonic stage (AFT) showed no dividing cells ( $n=7$ VNCs). Strikingly, in seq mutants we observed a number of dividing cells even at this late embryonic stage ( $n=10$ VNCs; Fig. 3G,H)

The Notch pathway is well known to play a crucial role in limiting NB selection during early neurogenesis (Poulson, 1937; Lehmann et al., 1983; Skeath and Thor, 2003). In addition, previous studies have revealed a connection between seq and the Notch pathway (Andrews et al., 2009). Therefore, the elevated proliferation observed in seq mutants could in theory result from the generation of supernumerary NBs. This prompted us to quantify NB numbers in seq mutants. However, using Dpn/Pros as markers to identify NBs, we did not observe any significant increase in NB numbers in seq mutants when compared with the control (Fig. 3I).

In line with the results obtained in the NB5-6T and NB3-3A lineages, seq mutants display a global failure to execute the type I $>0$ daughter proliferation switch. In addition, global analysis reveals a failure to execute precise NB cell cycle exit (Fig. 3J). Importantly, the proliferation increase in NBs or daughters in seq mutants is not due to the generation of supernumerary NBs during early neurogenesis.

\section{seq regulates three key cell cycle genes}

Our recent candidate gene analysis of proliferation control in the Drosophila VNC identified key roles for a handful of cell cycle

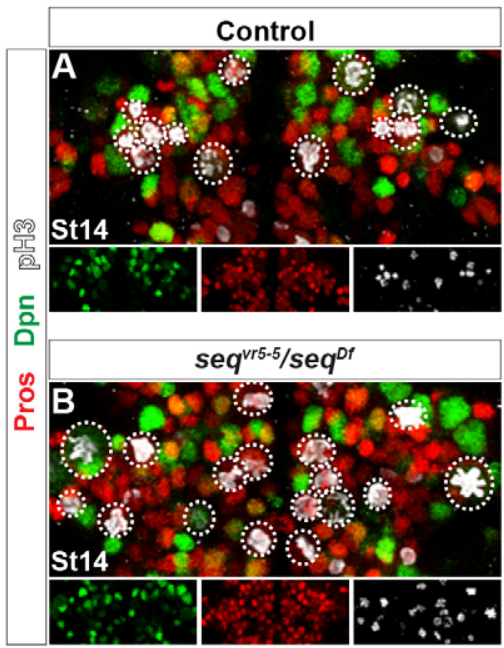

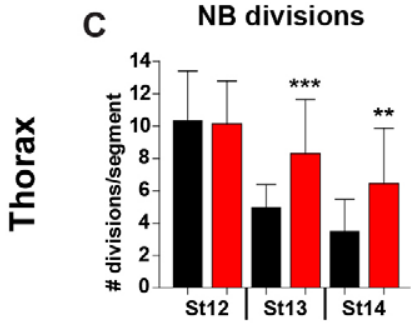

E

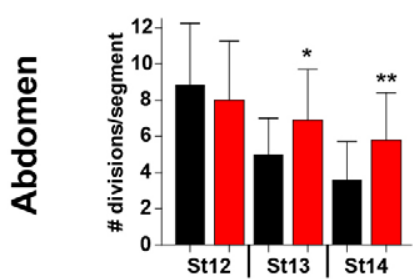

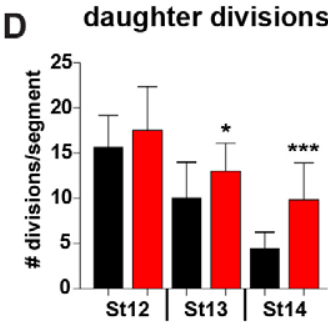

$\mathbf{F}$

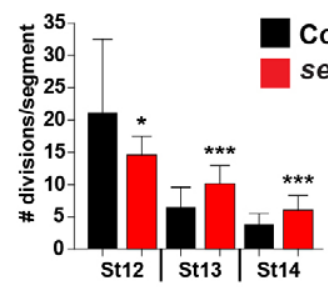

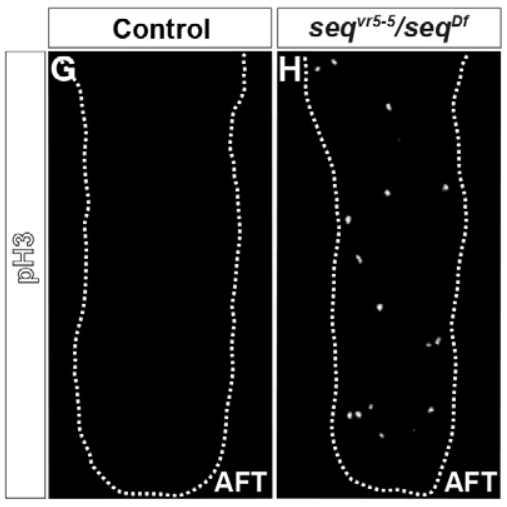

I

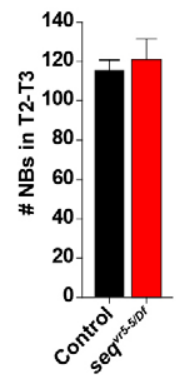

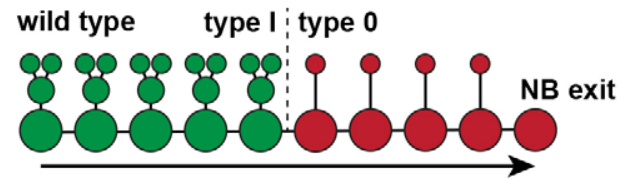

sequoia

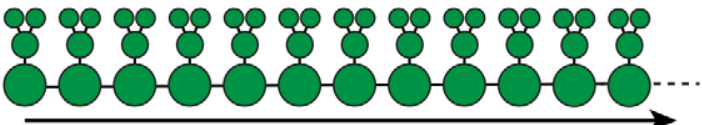

Fig. 3. seq mutants exhibit overproliferation globally. (A,B) Mitotic NBs and daughters in one thoracic segment, at St14, in control and seq mutants. Mitotic NBs are identifiable by being Dpn positive, Pros cortical asymmetric and $\mathrm{pH} 3$ positive, whereas mitotic daughters are identified by being Dpn negative, Pros nuclear and $\mathrm{pH} 3$ positive. (C-F) Quantification of dividing NBs and daughters in thorax and abdomen at St12, St13 and St14. ${ }^{*} P \leq 0.05,{ }^{* *} P \leq 0.01$, ${ }^{* * *} P \leq 0.001$, twotailed Student's $t$-test; $n=20$ segments for T2-T3 and A1-A2; error bars indicate s.d. Increased numbers of dividing NBs and daughters are observed in seq mutants during later stages in both thorax and abdomen. $(\mathrm{G}, \mathrm{H})$ Mitotic cells are never observed in control VNCs at late embryonic stage (AFT), whereas seq mutants display a number of mitotic cells ( $n \geq 7$ VNCs). (I) Quantification of NBs in T2-T3 does not reveal significantly altered NB numbers in seq mutants. Twotailed Student's $t$-test; $n=40$ thoracic hemisegments; error bars indicate s.d. (J) Diagram depicting that seq mutants display a failed type $1>0$ switch, as well as failure in NB exit. 
genes, in particular $C y c E, E 2 f 1$, stg and dap (Baumgardt et al., 2014). In addition, the $F M R F a-e G F P$ forward genetic screen specifically identified $C y c E, E 2 f 1$ and dap (Bivik et al., 2015). The strong proliferation effects observed in seq mutants prompted us to address whether seq is acting on these four key cell cycle genes. To quantify these effects, we stained with antibodies for CycE, E2f1, Stg or Dap in control and seq mutants at the same developmental stage and on the same slide, and scanned the VNCs using identical confocal microcopy imaging settings. Expression (mean pixel intensity) was measured globally in dividing thoracic NBs, and each dataset was normalized for the average expression in control NBs and for the volume of each NB measured (see Materials and Methods). These quantifications revealed significant upregulation of both $\mathrm{CycE}$ and E2f1, and a weaker upregulation of Dap (Fig. 4A-F,I), whereas Stg expression was unaffected (Fig. 4G,I). The upregulation of $\mathrm{CycE}$ and E2f1 was also apparent when analyzing the NB5-6T lineage specifically. By contrast, we noted downregulation of Dap (Fig. 4J).

The strong upregulation of $\mathrm{CycE}$, and the crucial role of $C y c E$ in lineage progression in the VNC (Baumgardt et al., 2014), prompted us to test whether $C y c E$ interacts genetically with seq using the supernumerary Eya cells generated in the NB5-6T lineage as readout. We removed one gene copy of $C y c E$ in a $s e q$ hemizygous background and indeed noted a significant reduction of supernumerary Eya cells (Fig. 4K).

We conclude that Seq represses three key cell cycle factors, namely CycE, E2F1 and Dap, and that the failed type I $>0$ daughter proliferation switch observed in seq mutants may, at least in part, be explained by elevated CycE expression.
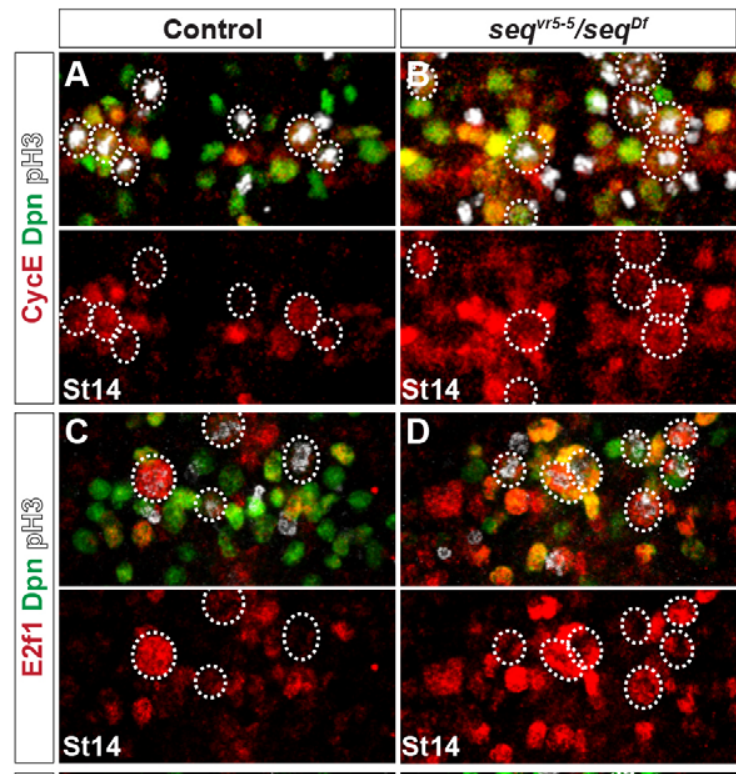

I
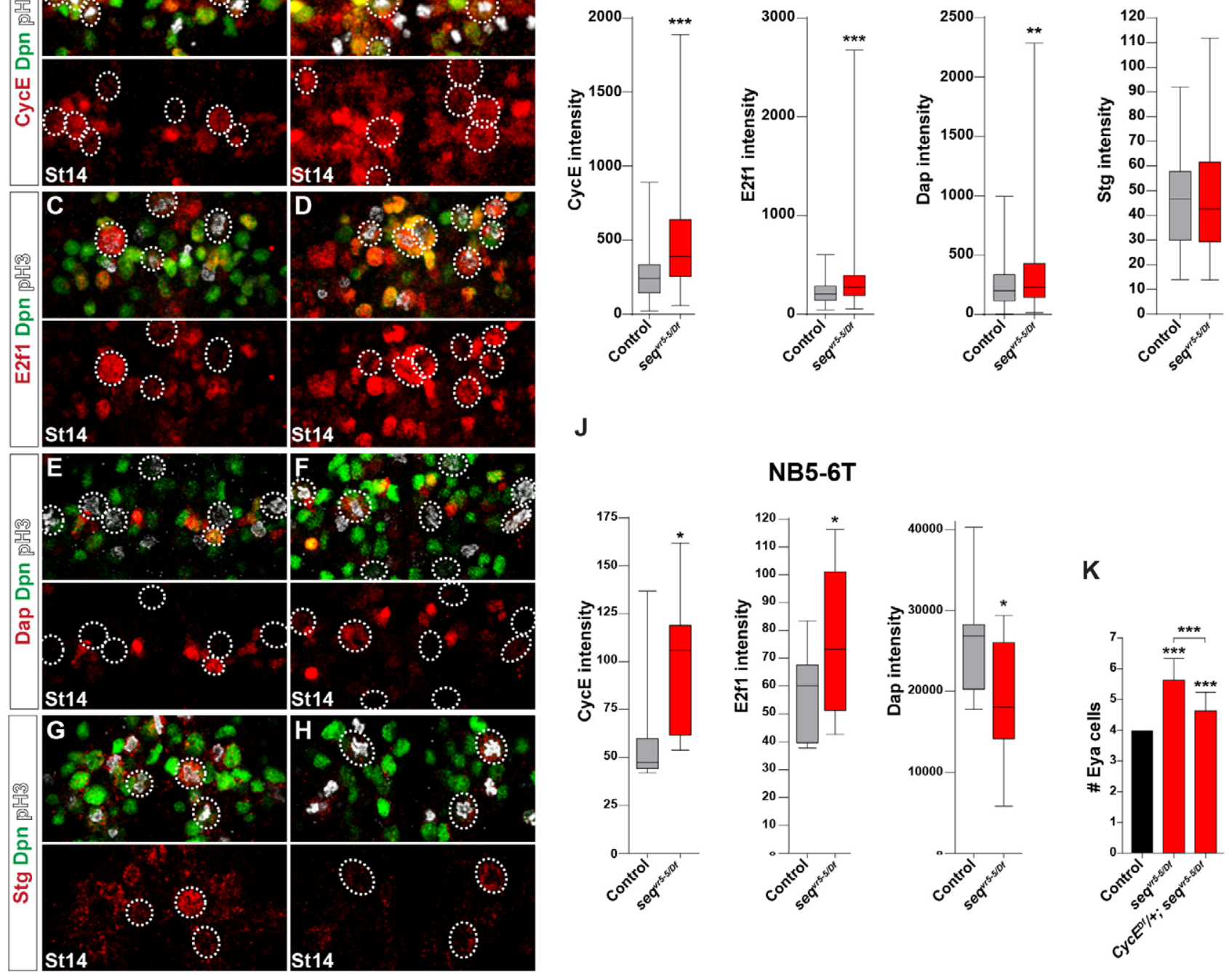

$J$



\section{NB5-6T}
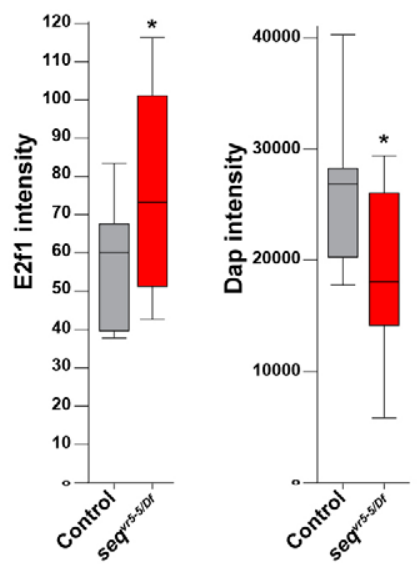

$\mathbf{K}$

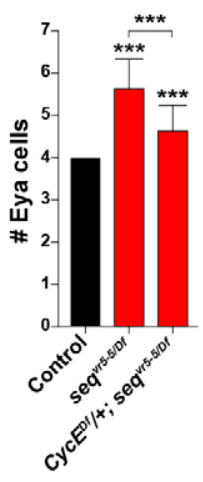

Fig. 4. seq mutants show elevated CycE and E2f1 expression. (A-H) Expression of the cell cycle proteins CycE, E2f1, Dap and Stg in NBs in control and seq mutants at St14. Expression of CycE, E2f1 and Dap appears upregulated in seq mutants, whereas Stg is not clearly affected. NBs are circled. (I,J) Quantification of CycE, E2f1, Dap and Stg protein levels by measurement of fluorescence intensity levels. (I) In thoracic NBs, CycE, E2f1 and Dap are significantly upregulated in seq mutants, with the strongest effect on CycE expression. (J) In NB5-6T, CycE and E2f1 are upregulated, whereas Dap is downregulated. (K) Quantification of the number of Eya cells in the NB5-6T lineage in control, seq mutants, and seq mutants heterozygous for CycE ${ }^{D f}$. Heterozygosity for CycE significantly suppresses the increase in Eya cell numbers in seq mutants. ${ }^{*} P \leq 0.05,{ }^{* *} P \leq 0.01$, ${ }^{* * *} P \leq 0.001$, two-tailed Student's $t$-test $(\mathrm{I}, \mathrm{J})$ or one-way ANOVA with Bonferroni multiple comparison (K); $n \geq 100$ (I) or $n \geq 11$ (J) NBs, $n \geq 40$ lineages (K); error bars indicate s.d. 


\section{seq interacts with the Notch signaling pathway}

Given the connections between seq, ttk, the PRDM family and Notch signaling, we next addressed the possible connection between seq and Notch signaling. We analyzed the expression of the Notch target gene and key effector $E$ (spl)m8-HLH (Jennings et al., 1994; Bang et al., 1995; Lecourtois and Schweisguth, $1995)$ using an $E(s p l) m 8-H L H-G F P$ reporter transgene that is known to be dependent upon Notch signaling in VNC NBs (Ulvklo et al., 2012; Bivik et al., 2016). In controls at St14, GFP expression was apparent in many, but not all NBs in the VNC (Fig. 5A). This is in agreement with previous studies of NB5-6T, which demonstrated a gradual activation of this Notch reporter from St12 onward (Ulvklo et al., 2012). By contrast, in seq mutants GFP expression was observed in significantly more NBs (Fig. 5A-C), with overall expression levels significantly elevated in NBs (Fig. 5D).

The temporal onset of $E(s p l) m 8-H L H-G F P$ expression in NB5$6 \mathrm{~T}$ (Ulvklo et al., 2012) and the apparent role of seq in controlling both onset and levels prompted us to address how Seq protein expression in NBs changes over time. We quantified Seq protein levels in control embryos in T1-T3 NBs at St12, St13 and St14 and noted a gradual and significant reduction of Seq levels over time (Fig. 5E). This gradual decline in Seq expression was also noted in NB5-6T, specifically (Fig. S1D,E). We also monitored Seq expression simultaneously with $E(\mathrm{spl}) m 8-H L H-G F P$ expression in thoracic NBs from St12-16. We observed a progression from most NBs expressing Seq alone at St12, to many NBs co-expressing Seq-GFP at St14, and the appearance of GFP-only NBs at St16 (Fig. S1A-C)

These expression results could indicate that Seq expression levels play a role in 'gating' the onset of Notch signaling. To further test this idea we overexpressed seq using the elav-Gal4 driver, which commences in NBs at St11 (Berger et al., 2007). However, overexpression of seq did not show a significant downregulation of $E(s p l) m 8-H L H-G F P$ expression when compared with the control (Fig. 5F). Surprisingly, overexpression of seq phenocopied seq
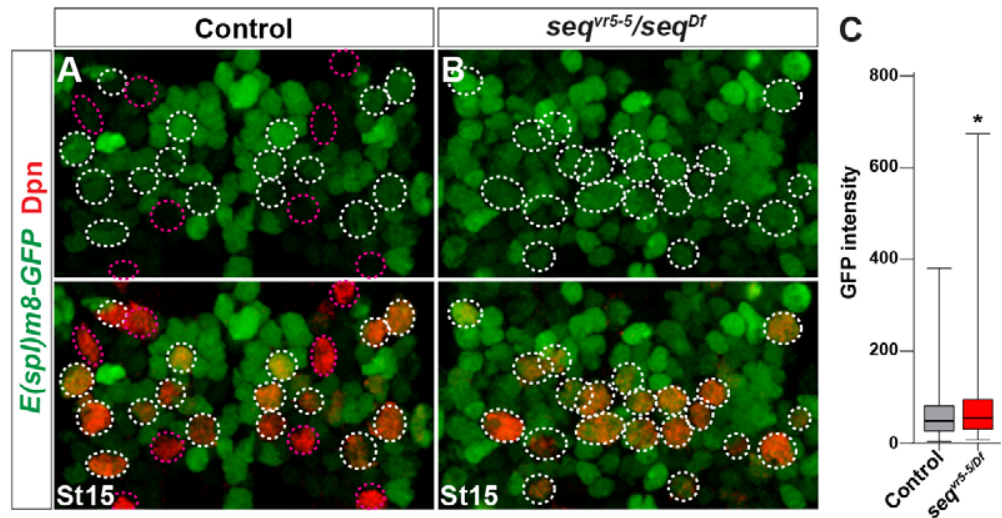



E

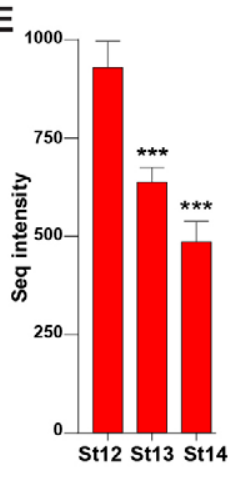

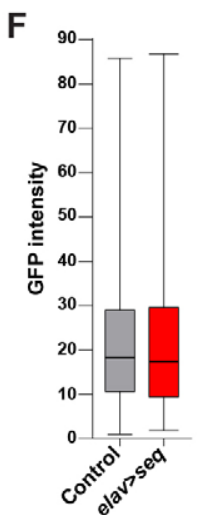

J

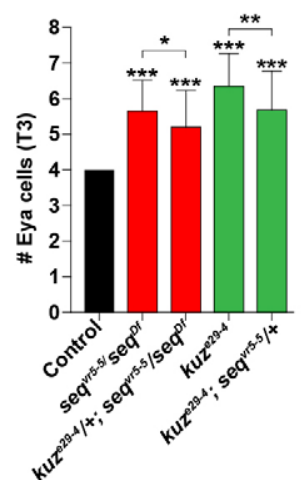

K

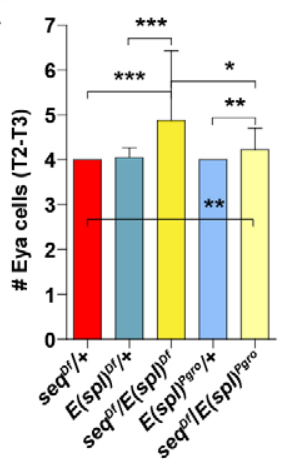

Fig. 5. seq regulates Notch signaling. (A,B) Expression of $E(s p l) H L H m 8-G F P$ in control and seq mutants. At St15 in control, some NBs robustly express GFP (white outlines), whereas others express GFP weakly or below detection (magenta outlines). By contrast, in seq mutants more NBs express GFP robustly.

(C) Quantification of the mean intensity pixels in NBs for $E(s p /) m 8-H L H-G F P$ in control and seq at St15 reveals significant upregulation of GFP in seq

(D) Quantification of the percentage of GFP-expressing NBs, based upon $E(s p l) m 8-H L H$-GFP, in control and seq at St15 reveals significant increase in the percentage of expressing NBs in seq. (E) Quantification of the mean intensity of pixels for Seq protein expression in control at St12, St13 and St14 reveals a gradual decrease in expression. (F) Quantification of the mean intensity of pixels for $E(s p /) m 8-H L H$-GFP expression in control versus seq overexpression at St15. seq overexpression does not result in significant downregulation of GFP levels. (G) Quantification of dividing NBs and daughters in thorax and abdomen at St13 in control and elav-Gal4/UAS-seq. Increased numbers of dividing NBs and daughters are observed when seq is overexpressed at St13 in both thorax and abdomen. (H) Expression of Seq protein in NBs in kuz (a participant in the Notch signaling pathway) mutants did not appear altered compared with control. (I) Quantification of the mean intensity of pixels for Seq protein expression in control and kuz mutants reveals no significant effect on Seq expression in kuz. (J) Quantification of the number of Eya cells in the NB5-6T lineage in control embryos, in seq homozygous mutants, kuz homozygous mutants, and seq or kuz homozygous mutants simultaneously heterozygous for seq or kuz. Heterozygosity for seq or kuz significantly suppresses the effects of seq or kuz homozygosity with respect to Eya cell numbers. (K) Quantification of the number of Ap cells in the NB5-6T lineage in heterozygotes for seq ${ }^{D f}[D f(2 R) E x e / 8057], E(s p l)^{D f}[D f(3 R) B S C 751]$ and $E(s p l)^{P g r o}$ [a deficiency with a gro rescue construct=Df(3R)gro32.2, $P$-gro], as well as in transheterozygotes at stage AFT. Neither seq ${ }^{D f}, E(s p l)^{D f}$ nor $E(s p l)^{P g r o}$ shows any heterozygotic effects on Ap cell numbers. By contrast, transheterozygotes significantly increased Ap cell numbers. ${ }^{*} P \leq 0.05$, ${ }^{* \star} P \leq 0.01$, ${ }^{* \star *} P \leq 0.001$, two-tailed Student's $t$-test; $n \geq 374$ (C), $n \geq 393$ (D), $n \geq 10$ (E), $n \geq 149$ (F) or $n \geq 142$ (I) NBs, $n \geq 10$ segments (G), $n \geq 42$ (J) or $n \geq 20$ (K) lineages; error bars indicate s.d. 
mutants and resulted in increased NB and daughter proliferation (Fig. 5G).

Next, we addressed if Notch signaling regulates Seq expression. We analyzed mutants in the kuz gene, which encodes an ADAM metalloprotease crucial for cleavage of the Notch receptor (Pan and Rubin, 1997; Sotillos et al., 1997), that have been demonstrated to reduce Notch signaling without affecting NB selection (Ulvklo et al., 2012; Bivik et al., 2016). However, we did not observe any apparent reduction in Seq protein levels in kuz mutants (Fig. 5H,I).

Because of the gradual decrease in Seq expression, the gradual increase in $E(\mathrm{spl}) m 8-H L H-G F P$ expression, and the role of seq in repressing $E(s p l) m 8-H L H-G F P$ expression, we postulated that the type $I>0$ daughter proliferation switch might be sensitive to genetic interaction between Notch signaling and seq. To test this we counted Ap cells in NB5-6T as readout for the type I $>0$ switch, and tested for interaction between seq and kuz. We found that the increased Ap cell numbers observed in seq and kuz homozygous mutants was significantly suppressed by simultaneous heterozygosity for $k u z$ or seq, respectively (Fig. 5J).

Next, we addressed interaction between seq and the $E(s p l) H L H$ Notch effectors using the $E(s p l)$ complex deficiency $D f(3 R)$ $B S C 751$, which removes all seven $E(s p l) H L H$ genes. Strikingly, although neither seq nor $E(s p l) H L H$ heterozygotes displayed any effect on Ap cell numbers, transheterozygotes showed an increase from the normal number of four Ap cells to almost six on average (Fig. 5K). This transheterozygous effect is similar in strength to that observed in the individual seq and kuz homozygous null mutants (Fig. 1J) (Ulvklo et al., 2012; Bivik et al., 2016). Analyzing cell divisions in NB5-6T, we found that whereas the control never showed any dividing daughters at St15 (Fig. 2B), we did observe aberrant daughter cell divisions in the seq/Df(3R)BSC751 transheterozygotes $(5 \%, n=40$ lineages). These results reveal that the increase in Ap cell numbers stems, at least in part, from a failed type $\mathrm{I}>0$ switch.

The $D f(3 R) B S C 751$ deletion also removes groucho (gro), a known transcriptional co-repressor for the E(spl)HLH regulators (Paroush et al., 1994; Fisher et al., 1996). Moreover, previous genetic analysis of the $E(s p l)$ complex indicated that gro is involved in the type I $>0$ switch (Bivik et al., 2016). To address the role of gro with respect to the interaction with $s e q$, we crossed seq to a deficiency line containing a gro rescue construct $[D f(3 R)$ gro32.2, $P$-gro]. This resulted in a significantly reduced effect upon Ap cell numbers when compared with seq $/ D f(3 R) B S C 751$ (Fig. 5K). However, there was still a significant increase in Ap cell numbers compared with seq or $D f(3 R)$ gro32.2, P-gro heterozygotes (Fig. 5K). From these findings we conclude that seq interacts with both the $E(s p l) H L H$ and gro genes.

\section{DISCUSSION}

seq controls the type I>0 daughter proliferation switch; a balancing act with Notch signaling?

In seq mutants we find an increase in the number of cells in the NB5$6 \mathrm{~T}$ and NB3-3A lineages, as well as aberrant daughter divisions in both lineages. This effect is mirrored globally, with elevated daughter divisions in both the thorax and abdomen. From these results we conclude that seq plays a key role in promoting the type $\mathrm{I}>0$ daughter proliferation switch. Surprisingly, we find that seq overexpression also triggers aberrant type $I>0$ switches. These results could indicate that Seq expression levels are instructive, with high levels promoting the type I proliferation mode and lower levels promoting type 0 . On that note, there is precedence for transcription factors switching between repressor and activator function in a concentration-dependent manner (Sauer and Jackle, 1991, 1993; Bourguignon et al., 1998; Wallin et al., 1998). We also observe elevated NB proliferation in seq mutants and with seq overexpression, in NB3-3A and globally, indicating that the precise NB cell cycle exit at the end of lineage progression is affected by seq function and levels. We find that seq represses $\mathrm{CycE}$ and E2f1, both in thoracic NBs in general and specifically in NB56T. Dap expression was weakly increased globally, but weakly reduced in NB5-6T, suggesting an aspect of context dependency with respect to seq regulation of Dap.

Notch signaling also plays a key role in triggering the type $\mathrm{I}>0$ switch, and Notch reporter [E(spl)HLHm8-GFP] expression is turned on in NBs during the latter stages of lineage progression (Ulvklo et al., 2012; Bivik et al., 2016). This indicates that the timing of Notch signaling onset is important for the precision of the type $\mathrm{I}>0$ switch. Notch activation results in the formation of a tripartite protein complex comprising the Notch intracellular domain (NICD), the DNA-binding factor $\mathrm{Su}(\mathrm{H})$ and the co-factor Mastermind (Mam) (Kopan and Ilagan, 2009; Hori et al., 2013). Molecular and genetic in-depth analysis of how Notch signaling controls the type $\mathrm{I}>0$ switch points to a multi-level model whereby $\mathrm{NICD} / \mathrm{Su}(\mathrm{H}) / \mathrm{Mam}$ activates $E(\mathrm{spl}) H L H$ and dap, and $\mathrm{E}(\mathrm{spl}) \mathrm{HLH}$ subsequently represses $C y c E, E 2 f 1$ and stg (Bivik et al., 2016).

Our findings point to a complex balancing interplay between seq, the Notch pathway and the cell cycle. We observe premature and elevated Notch reporter $[E(s p l) H L H m 8-G F P]$ expression in seq mutants, indicating that seq represses Notch signaling in NBs. However, seq mutants phenocopy Notch pathway perturbation, and both result in a failure to execute the type I $>0$ switch. Moreover, seq and $E(s p l) H L H$ interact strongly genetically in transheterozygotes, as is evident by increased Ap cell numbers. Based on these findings, we propose that Seq acts at several steps of the Notch type $\mathrm{I}>0$ cascade by repressing not only $E(s p l) H L H$ but also $C y c E$ and $E 2 f 1$, and that the balance in regulation of these different targets is sensitive to the levels of Seq. Specifically, we propose that Seq is a stronger repressor of $C y c E$ and $E 2 f l$ than of $E(s p l) H L H$. Combined with the repressive role of $\mathrm{E}(\mathrm{spl}) \mathrm{HLH}$ on $C y c E$ and $E 2 f 1$, this model might also help to explain why seq mutants phenocopy seq overexpression.

\section{Seq acts in a context-dependent manner in relation to Notch signaling}

Notch signaling plays a key role during development of the external sensory organs (ESOs) in Drosophila (Dietrich and Campos-Ortega, 1984; Hartenstein and Posakony, 1989; Simpson, 1990). Notch acts at multiple steps of ESO development: in the process of lateral inhibition, to select the sensory organ precursor (SOP), and during subsequent asymmetric cell division events in the SOP lineage (reviewed by Schweisguth, 2015). Previous studies of seq in the developing ESOs revealed defects both in the external lineage, with a conversion of shaft cells into socket cells, and the internal lineage, with loss of both neuron and glia specification. These phenotypes could to some extent be explained by the loss of expression of three Notch target genes: D-Pax2 (shaven - FlyBase), pros and hamlet (ham) (Andrews et al., 2009). Interestingly, of these three seq targets, only ham was affected in the embryonic PNS (Andrews et al., 2009). Comparing the role of Notch signaling with that of seq during ESO development, in spite of sharing several target genes seq only partly phenocopies Notch pathway mutants: Notch clones show supernumerary SOP cells and conversion of the entire SOP lineage into neurons, whereas seq clones do not show extra SOP cells but instead shaft-to-socket conversion and neuron/glia specification defects. 
Similar to the lack of SOP selection effects in ESOs in seq mutants, we did not observe any effects upon NB selection in the embryonic neuroectoderm, a process also controlled by Notch-mediated lateral inhibition. Instead, seq acts at a later stage to modulate expression of the Notch targets $E(s p l) H L H$ and $C y c E$, during the subsequent type $\mathrm{I}>0$ daughter proliferation switch.

These studies reveal that the interplay between seq and the Notch pathway is highly context dependent, and seq appears to act on different Notch subroutines in different settings. We also observe elevated NB divisions in seq mutants, in NB3-3A and globally. This is in contrast to Notch signaling, which does not appear to affect NB cell cycle exit in the VNC (Ulvklo et al., 2012; Bivik et al., 2016). Hence, seq can also play roles that are independent of Notch signaling during nervous system development.

\section{Seq, Ttk and the PRDM family: an expanding group of Notch interactors}

The $\mathrm{C} 2 \mathrm{H} 2$ zinc fingers of Seq are highly homologous to the zinc fingers in Ttk (Brenman et al., 2001). Seq and Ttk furthermore share homology in their zinc fingers with members of the PRDM family (Hohenauer and Moore, 2012). In addition to related zinc fingers, Seq, Ttk and the PRDM family also share an involvement in Notch pathway signaling. $t t k$ has been identified as a Notch pathway target gene and effector, both during oogenesis and ESO development (Guo et al., 1996; Althauser et al., 2005; Jordan et al., 2006). The PRDM family plays important roles during development and is also intimately linked to Notch signaling (Hohenauer and Moore, 2012; Zannino and Sagerstrom, 2015). The Drosophila ham gene, a PRDM family member, was found to control ESO development, chiefly by modulating Notch signaling (Moore et al., 2002, 2004). Similarly, in olfactory sensory lineages, ham also modifies Notch signaling, intriguingly by repressing $E(s p l) H L H$ gene expression via direct binding of Ham to this complex (Endo et al., 2011). In the developing mammalian CNS, $\operatorname{Prdm} 8$ and $\operatorname{Prdm} 16$ were found to be regulated by the bHLH HES genes Hes 1, Hes 3 and Hes 5 (Kinameri et al., 2008).

Dynamic protein expression is another common denominator. Seq, Ttk and Ham were all found to be dynamically expressed in the developing SOPs (Moore et al., 2002, 2004; Andrews et al., 2009; Endo et al., 2011). In addition, dynamic Seq protein expression levels govern photoreceptor axon targeting to the optic lobe (Petrovic and Hummel, 2008; Kulkarni et al., 2016).

In summary, Seq, Ttk and the PRDM family have in common their intimate connection to the Notch pathway, acting to regulate the pathway, and/or being regulated by it, and/or regulating Notch downstream targets. They also share the property of controlling cell fate and proliferation in the developing nervous system, in some cases acting on the same targets (e.g. CycE) (Badenhorst, 2001; Brumby et al., 2004). Finally, they are highly dynamic in their expression, and the expression levels can act in instructive and temporal manners. Our results for seq support the role of the extended PRDM family as context-dependent, temporally controlled and level-sensitive modifiers of Notch signaling during nervous system development.

\section{MATERIALS AND METHODS \\ Fly stocks}

lbe(K)-EGFP (Ulvklo et al., 2012). lbe(K)-lacZ (Baumgardt et al., 2014). elav-Gal4 (DiAntonio et al., 2001). FMRFa-eGFP, seq ${ }^{03 F H}$ and FMRFa-eGFP (Bivik et al., 2015). UAS-nls-myc-EGFP (referred to as
UAS-nmEGFP) (Allan et al., 2003). E(spl)m8-EGFP (Castro et al., 2005) (provided by Jim Posakony). $D f(3 R)$ gro 32.2, P-gro (provided by Francois Schweisguth, Institute Pasteur, Paris, France). From the Bloomington Drosophila Stock Center: kuz $\mathrm{z}^{29-4}$ (\#5804), E(spl) complex deficiency $=D f$ (3R)BSC751 (\#26849), UAS-seq (\#9244), CycE $E^{D f}=D f(2 L)$ Exel7063 (\#7831); for apoptosis deletion: Df(3L)ED225 (\#8081), seq $q^{v r 5-5}$ (\#5559), seq $^{D f}=D f(2 R)$ Exel8057 (\#7871), e $g^{M z 360}$ (referred to as e $g^{\text {Gal4 }}$; \#8758). Mutants were maintained over $G F P$ - or $Y F P$-marked balancer chromosomes. As control, OregonR or $w^{1118}$ was used. Staging of embryos was performed according to Campos-Ortega and Hartenstein (1985).

\section{Immunohistochemistry}

Immunohistochemistry was performed as previously described (Baumgardt et al., 2009) using the following primary antibodies. Guinea pig anti-Dpn (1:1000) and rat anti-Dpn (1:500) (Ulvklo et al., 2012). Rabbit and rat antiphospho-Histone H3 Ser10 (pH3) (1:250; Upstate/Millipore, 06-570). Rabbit anti-GFP (1:1000) (Molecular Probes, A-21311) and chicken antiGFP (1:1000) (Abcam, ab13979). Chicken anti-proNplp1 (1:1000) and rabbit anti-proFMRFa (1:1000) (Baumgardt et al., 2007). Chicken anti- $\beta$ Gal (1:1000; Abcam, ab9361). Rabbit anti-Seq (1:1000) (Brenman et al., 2001) (provided by Yuh-Nung Jan). Mouse monoclonal antibodies antiPros MR1A (1:10), anti-Eya 10H6 (1:250) and anti-Eve $(1: 10)$ (Developmental Studies Hybridoma Bank). Rabbit anti-CycE (1:500; Santa Cruz Biotechnology, SC-33748). Guinea pig anti-Dap (1:1000) and rat anti-E2f1 (1:100) (Baumgardt et al., 2014). Rat anti-Stg (1:500) (Bivik et al., 2016)

\section{Confocal imaging and data acquisition}

For fluorescent images a Zeiss LSM 700 confocal microscope was used, with LSM Image Browser software (v4.2) or Adobe Photoshop (CS6) for merging confocal stacks, and Adobe Illustrator (CS6) for compiling images. For statistical calculations GraphPad Prism (v6.05) was used, and graphs were compiled in Adobe Illustrator (CS6).

\section{Statistical analysis}

GraphPad Prism (v6.05) was used for all statistical calculations. To test statistical significance between two normally distributed groups, Student's $t$-test was used. When comparing more than two groups, ANOVA and Bonferroni multiple comparison were used. For analysis of proliferation frequencies in the lineages NB3-3A and NB5-6T, Chi-square was used. Evaluation of expression levels was performed by dissecting the CNS from mutant and control embryos on the same slide in at least two different experiments. The intensity of staining in NBs was measured in Adobe Photoshop (CS6) or in ImageJ (1.49d). To compare the results from separate experiments, the means were normalized and multiplied by volume or area in Adobe Photoshop or ImageJ, respectively, to compensate for size To quantify differences on the whole dataset, Student's $t$-test was performed.

\section{Acknowledgements}

We are grateful to Yuh-Nung Jan, Hugo Bellen, Jim Posakony, Francois Schweisguth, the Developmental Studies Hybridoma Bank at the University of lowa, and the Bloomington Stock Center for sharing antibodies, fly lines and DNAs. We thank Don van Meyel for critically reading the manuscript. Helen Ekman and Carolin Jonsson provided excellent technical assistance.

\section{Competing interests}

The authors declare no competing or financial interests.

\section{Author contributions}

A.S. mapped the seq ${ }^{03 F H}$ allele. E.G. and C.B. performed all other experiments. E.G., C.B. and S.T. performed the statistical analysis. E.G. and S.T. compiled the figures. E.G. and S.T. wrote the manuscript, with contributions from C.B.

\section{Funding}

This work was supported by the Swedish Research Council (Vetenskapsrådet), the Knut and Alice Wallenberg Foundation (Knut och Alice Wallenbergs Stiftelse) and the Swedish Cancer Foundation (Cancerfonden) (S.T.) 
Supplementary information

Supplementary information available online at

http://dev.biologists.org/lookup/doi/10.1242/dev.139998.supplemental

\section{References}

Allan, D. W., Pierre, S. E. S., Miguel-Aliaga, I. and Thor, S. (2003). Specification of neuropeptide cell identity by the integration of retrograde BMP signaling and a combinatorial transcription factor code. Cell 113, 73-86.

Althauser, C., Jordan, K. C., Deng, W.-M. and Ruohola-Baker, H. (2005). Fringedependent notch activation and tramtrack function are required for specification of the polar cells in Drosophila oogenesis. Dev. Dyn. 232, 1013-1020.

Andrews, H. K., Giagtzoglou, N., Yamamoto, S., Schulze, K. L. and Bellen, H. J. (2009). Sequoia regulates cell fate decisions in the external sensory organs of adult Drosophila. EMBO Rep. 10, 636-641.

Badenhorst, P. (2001). Tramtrack controls glial number and identity in the Drosophila embryonic CNS. Development 128, 4093-4101.

Bang, A. G., Bailey, A. M. and Posakony, J. W. (1995). Hairless promotes stable commitment to the sensory organ precursor cell fate by negatively regulating the activity of the Notch signaling pathway. Dev. Biol. 172, 479-494.

Baumgardt, M., Miguel-Aliaga, I., Karlsson, D., Ekman, H. and Thor, S. (2007) Specification of neuronal identities by feedforward combinatorial coding. PLoS Biol. 5, e37.

Baumgardt, M., Karlsson, D., Terriente, J., Díaz-Benjumea, F. J. and Thor, S. (2009). Neuronal subtype specification within a lineage by opposing temporal feed-forward loops. Cell 139, 969-982.

Baumgardt, M., Karlsson, D., Salmani, B. Y., Bivik, C., MacDonald, R. B. Gunnar, E. and Thor, S. (2014). Global programmed switch in neural daughter cell proliferation mode triggered by a temporal gene cascade. Dev. Cell 30, 192-208

Bello, B. C., Izergina, N., Caussinus, E. and Reichert, H. (2008). Amplification of neural stem cell proliferation by intermediate progenitor cells in Drosophila brain development. Neural Dev. 3, 5

Berger, C., Renner, S., Lüer, K. and Technau, G. M. (2007). The commonly used marker ELAV is transiently expressed in neuroblasts and glial cells in the Drosophila embryonic CNS. Dev. Dyn. 236, 3562-3568.

Bertet, C., Li, X., Erclik, T., Cavey, M., Wells, B. and Desplan, C. (2014). Tempora patterning of neuroblasts controls Notch-mediated cell survival through regulation of Hid or Reaper. Cell 158, 1173-1186.

Birkholz, O., Rickert, C., Berger, C., Urbach, R. and Technau, G. M. (2013) Neuroblast pattern and identity in the Drosophila tail region and role of doublesex in the survival of sex-specific precursors. Development 140, 1830-1842.

Bivik, C., Bahrampour, S., Ulvklo, C., Nilsson, P., Angel, A., Fransson, F., Lundin, E., Renhorn, J. and Thor, S. (2015). Novel genes involved in controlling specification of Drosophila FMRFamide neuropeptide cells. Genetics 200, 1229-1244

Bivik, C., MacDonald, R. B., Gunnar, E., Mazouni, K., Schweisguth, F. and Thor S. (2016). Control of neural daughter cell proliferation by multi-level Notch/Su(H)/E (spl)-HLH signaling. PLoS Genet. 12, e1005984.

Boone, J. Q. and Doe, C. Q. (2008). Identification of Drosophila type II neuroblast lineages containing transit amplifying ganglion mother cells. Dev. Neurobiol. 68 $1185-1195$

Bossing, T., Udolph, G., Doe, C. Q. and Technau, G. M. (1996). The embryonic central nervous system lineages of Drosophila melanogaster. I. Neuroblast lineages derived from the ventral half of the neuroectoderm. Dev. Biol. 179, 41-64.

Bourguignon, C., Li, J. and Papalopulu, N. (1998). XBF-1, a winged helix transcription factor with dual activity, has a role in positioning neurogenesis in Xenopus competent ectoderm. Development 125, 4889-4900.

Bowman, S. K., Rolland, V., Betschinger, J., Kinsey, K. A., Emery, G. and Knoblich, J. A. (2008). The tumor suppressors Brat and Numb regulate transitamplifying neuroblast lineages in Drosophila. Dev. Cell 14, 535-546.

Brenman, J. E., Gao, F.-B., Jan, L. Y. and Jan, Y. N. (2001). Sequoia, a tramtrackrelated zinc finger protein, functions as a pan-neural regulator for dendrite and axon morphogenesis in Drosophila. Dev. Cell 1, 667-677.

Brumby, A., Secombe, J., Horsfield, J., Coombe, M., Amin, N., Coates, D., Saint R. and Richardson, H. (2004). A genetic screen for dominant modifiers of a cyclin $\mathrm{E}$ hypomorphic mutation identifies novel regulators of S-phase entry in Drosophila. Genetics 168, 227-251.

Campos-Ortega, J. A. and Hartenstein, V. (1985). The Embryonic Development of Drosophila Melanogaster. New York: Springer-Verlag.

Castro, B., Barolo, S., Bailey, A. M. and Posakony, J. W. (2005). Lateral inhibition in proneural clusters: cis-regulatory logic and default repression by Suppressor of Hairless. Development 132, 3333-3344.

DiAntonio, A., Haghighi, A. P., Portman, S. L., Lee, J. D., Amaranto, A. M. and Goodman, C. S. (2001). Ubiquitination-dependent mechanisms regulate synaptic growth and function. Nature 412, 449-452.

Dietrich, U. and Campos-Ortega, J. A. (1984). The expression of neurogenic loci in imaginal epidermal cells of Drosophila melanogaster. J. Neurogenet. 1, 315-332.
Endo, K., Karim, M. R., Taniguchi, H., Krejci, A., Kinameri, E., Siebert, M., Ito, K. Bray, S. J. and Moore, A. W. (2011). Chromatin modification of Notch targets in olfactory receptor neuron diversification. Nat. Neurosci. 15, 224-233.

Fish, J. L., Dehay, C., Kennedy, H. and Huttner, W. B. (2008). Making bigge brains-the evolution of neural-progenitor-cell division. J. Cell Sci. 121, 2783-2793.

Fisher, A. L., Ohsako, S. and Caudy, M. (1996). The WRPW motif of the hairyrelated basic helix-loop-helix repressor proteins acts as a 4-amino-acid transcription repression and protein-protein interaction domain. Mol. Cell. Biol. 16, $2670-2677$

Franco, S. J. and Müller, U. (2013). Shaping our minds: stem and progenitor cell diversity in the mammalian neocortex. Neuron 77, 19-34.

Gao, F. B., Brenman, J. E., Jan, L. Y. and Jan, Y. N. (1999). Genes regulating dendritic outgrowth, branching, and routing in Drosophila. Genes Dev. 13, 2549-2561.

Götz, M. and Huttner, W. B. (2005). The cell biology of neurogenesis. Nat. Rev. Mol. Cell Biol. 6, 777-788.

Guo, M., Jan, L. Y. and Jan, Y. N. (1996). Control of daughter cell fates during asymmetric division: interaction of Numb and Notch. Neuron 17, 27-41.

Hartenstein, V. and Posakony, J. W. (1989). Development of adult sensilla on the wing and notum of Drosophila melanogaster. Development 107, 389-405.

Hohenauer, T. and Moore, A. W. (2012). The Prdm family: expanding roles in stem cells and development. Development 139, 2267-2282.

Hori, K., Sen, A. and Artavanis-Tsakonas, S. (2013). Notch signaling at a glance. J. Cell Sci. 126, 2135-2140.

Jennings, B., Preiss, A., Delidakis, C. and Bray, S. (1994). The Notch signalling pathway is required for Enhancer of split bHLH protein expression during neurogenesis in the Drosophila embryo. Development 120, 3537-3548.

Jordan, K. C., Schaeffer, V., Fischer, K. A., Gray, E. E. and Ruohola-Baker, H. (2006). Notch signaling through tramtrack bypasses the mitosis promoting activity of the JNK pathway in the mitotic-to-endocycle transition of Drosophila follicle cells. BMC Dev. Biol. 6, 16.

Karlsson, D., Baumgardt, M. and Thor, S. (2010). Segment-specific neuronal subtype specification by the integration of anteroposterior and temporal cues. PLoS Biol. 8, e1000368.

Kinameri, E., Inoue, T., Aruga, J., Imayoshi, I., Kageyama, R., Shimogori, T. and Moore, A. W. (2008). Prdm proto-oncogene transcription factor family expression and interaction with the Notch-Hes pathway in mouse neurogenesis. PLOS ONE 3, e3859.

Kopan, R. and Ilagan, M. X. G. (2009). The canonical Notch signaling pathway: unfolding the activation mechanism. Cell 137, 216-233.

Kriegstein, A., Noctor, S. and Martínez-Cerdeno, V. (2006). Patterns of neural stem and progenitor cell division may underlie evolutionary cortical expansion. Nat. Rev. Neurosci. 7, 883-890.

Kulkarni, A., Ertekin, D., Lee, C.-H. and Hummel, T. (2016). Birth order dependent growth cone segregation determines synaptic layer identity in the Drosophila visual system. Elife 5, e13715.

Lecourtois, M. and Schweisguth, F. (1995). The neurogenic suppressor of hairless DNA-binding protein mediates the transcriptional activation of the enhancer of split complex genes triggered by Notch signaling. Genes Dev. 9, 2598-2608.

Lehmann, R., Jimenez, F., Dietrich, U. and Campos-Ortega, J. A. (1983). On the phenotype and development of mutants of early neurogenesis in Drosophila melanogaster. Roux's Arch. Dev. Biol. 192, 62-74.

Moore, A. W., Jan, L. Y. and Jan, Y. N. (2002). hamlet, a binary genetic switch between single- and multiple- dendrite neuron morphology. Science 297, 1355-1358.

Moore, A. W., Roegiers, F., Jan, L. Y. and Jan, Y. N. (2004). Conversion of neurons and glia to external-cell fates in the external sensory organs of Drosophila hamle mutants by a cousin-cousin cell-type respecification. Genes Dev. 18, 623-628.

Nonaka-Kinoshita, M., Reillo, I., Artegiani, B., Martínez-Martínez, M. A., Nelson, M., Borrell, V. and Calegari, F. (2013). Regulation of cerebral cortex size and folding by expansion of basal progenitors. EMBO J. 32, 1817-1828.

Pan, D. and Rubin, G. M. (1997). Kuzbanian controls proteolytic processing of Notch and mediates lateral inhibition during Drosophila and vertebrate neurogenesis. Cell 90, 271-280.

Paroush, Z., Finley, R. L., Jr, Kidd, T., Wainwright, S. M., Ingham, P. W., Brent, R. and Ish-Horowicz, D. (1994). Groucho is required for Drosophila neurogenesis segmentation, and sex determination and interacts directly with hairy-related bHLH proteins. Cell 79, 805-815.

Petrovic, M. and Hummel, T. (2008). Temporal identity in axonal target laye recognition. Nature 456, 800-803.

Poulson, D. F. (1937). Chromosomal deficiencies and the embryonic development of drosophila melanogaster. Proc. Natl. Acad. Sci. USA 23, 133-137.

Rogulja-Ortmann, A., Luer, K., Seibert, J., Rickert, C. and Technau, G. M. (2007). Programmed cell death in the embryonic central nervous system of Drosophila melanogaster. Development 134, 105-116.

Sauer, F. and Jäckle, H. (1991). Concentration-dependent transcriptional activation or repression by Krüppel from a single binding site. Nature 353, 563-566.

Sauer, F. and Jäckle, H. (1993). Dimerization and the control of transcription by Krüppel. Nature 364, 454-457. 
Schmid, A., Chiba, A. and Doe, C. Q. (1999). Clonal analysis of Drosophila embryonic neuroblasts: neural cell types, axon projections and muscle targets. Development 126, 4653-4689.

Schmidt, H., Rickert, C., Bossing, T., Vef, O., Urban, J. and Technau, G. M. (1997). The embryonic central nervous system lineages of Drosophila melanogaster. II. Neuroblast lineages derived from the dorsal part of the neuroectoderm. Dev. Biol. 189, 186-204.

Schweisguth, F. (2015). Asymmetric cell division in the Drosophila bristle lineage: from the polarization of sensory organ precursor cells to Notch-mediated binary fate decision. Wiley Interdiscip. Rev. Dev. Biol. 4, 299-309.

Simpson, P. (1990). Lateral inhibition and the development of the sensory bristles of the adult peripheral nervous system of Drosophila. Development 109, 509-519.

Skeath, J. B. and Thor, S. (2003). Genetic control of Drosophila nerve cord development. Curr. Opin. Neurobiol. 13, 8-15.

Sotillos, S., Roch, F. and Campuzano, S. (1997). The metalloprotease-disintegrin Kuzbanian participates in Notch activation during growth and patterning of Drosophila imaginal discs. Development 124, 4769-4779.

Sousa-Nunes, R. and Somers, W. G. (2013). Mechanisms of asymmetric progenitor divisions in the Drosophila central nervous system. Adv. Exp. Med. Biol. 786, 79-102.
Ulvklo, C., MacDonald, R., Bivik, C., Baumgardt, M., Karlsson, D. and Thor, S. (2012). Control of neuronal cell fate and number by integration of distinct daughter cell proliferation modes with temporal progression. Development 139, 678-689.

Urbach, R., Schnabel, R. and Technau, G. M. (2003). The pattern of neuroblast formation, mitotic domains and proneural gene expression during early brain development in Drosophila. Development 130, 3589-3606.

Urbach, R., Jussen, D. and Technau, G. M. (2016). Gene expression profiles uncover individual identities of gnathal neuroblasts and serial homologies in the embryonic CNS of Drosophila. Development 143, 1290-1301.

Wallin, J. J., Gackstetter, E. R. and Koshland, M. E. (1998). Dependence of BSAP repressor and activator functions on BSAP concentration. Science $\mathbf{2 7 9}$, 1961-1964.

Wheeler, S. R., Stagg, S. B. and Crews, S. T. (2009). MidExDB: a database of Drosophila CNS midline cell gene expression. BMC Dev. Biol. 9, 56.

Zannino, D. A. and Sagerström, C. G. (2015). An emerging role for prdm family genes in dorsoventral patterning of the vertebrate nervous system. Neural Dev. 10, 24. 